\title{
Effect of Various Excipients on Theophylline-loaded Alginate Beads Prepared by lonic Cross Linking Technique
}

\author{
Sheikh Tasnim J ahan, Sams Mohammad Anowar Sadat, Md. Saiful Islam, \\ Reza-ul J alil and J akir Ahmed Chowdhury
}

\author{
Department of Pharmaceutical Technology, Faculty of Pharmacy, University of Dhaka, \\ Dhaka-1000, Bangladesh
}

\begin{abstract}
Theophylline loaded sodium alginate beads were prepared by ionic cross linking technique using calcium chloride $\left(\mathrm{CaCl}_{2}\right)$ as cross linking agents. The purpose of this work was to prepare sodium alginate beads as a device for the extended release of theophylline. Different excipients like sodium carboxy methyl cellulose, polyethylene glycol 4000, hydroxy propyl methyl cellulose, sodium starch glycolate, Eudragit L 100 and sodium lauryl sulphate were used to fabricate theophylline-alginate beads and their effect on drug release were investigated. In this study, the beads were characterized and evaluated in respect to their surface morphology, swelling index (SI) and in-vitro release characteristics. Beads were prepared by dropping a hot aqueous theophylline-alginate or theophylline-alginate-excipient solution into electrolyte solution. Alginate cross linked with electrolytes and beads were formed with entrapped drug. Beads were collected by decanting the solution and dried at room temperature. Surface of beads with various excipients revealed that smooth, dense and closely packed drug-polymer bonding was obtained when the excipients were changed. Beads in F 1 contain Eudragit L 100 that swelled highest at 3 hours with SI of $10.74 \%$. Sodium starch glycolate beads (F 4) swelled high up to $9.93 \%$ at 2 hours. Dissolution studies were carried out in $900 \mathrm{ml}$ of distilled water for 8 hours. Most of the formulations were fitted to Higuchi model. The drug release rate are shown in decreasing order: Eudragit L 100 $>$ Sodium carboxy methyl cellulose $>$ Sodium lauryl sulphate $>$ Sodium starch glycolate $>$ Hydroxy propyl methylcellulose $5 \mathrm{cps}>$ Polyethylene glycol 4000 . The use of Eudragit L 100 was found to be promising because it released about $69 \%$ of theophylline within 8 hours. It was found that among the hydrophilic polymers used, Sodium carboxy methyl cellulose showed $49 \%$ theophylline release within 8 hours. The lowest amount of drug release was found with HPMC 5 cps and PEG 4000 which was about $26 \%$ of drug release.
\end{abstract}

Key words: Theophylline, ionic cross-linking technique, sodium alginate beads, swelling index, release kinetics.

\section{INTRODUCTION}

Theophylline is a bronchodilator, which is used in treating Chronic Obstructive Pulmonary Disease (COPD). Conventional dosage forms of theophylline are administered 3-4 times a day to avoid large fluctuations in plasma concentrations. It is rapidly absorbed and eliminated. Our aim was to develop a sustained release preparation of theophylline to provide desirable serum

Correspondence to:

Jakir Ahmed Chowdhury

Tel: +880-2-7219747; Fax: +880-2-8615583

E-mail: jakir@univdhaka.edu

Dhaka Univ. J. Pharm. Sci. 9(1): 15-22, 2010 (June) concentrations for prolonged periods without frequent dosing, thereby providing patient compliance. ${ }^{1}$

The main concern of this study was to prepare good sustained release sodium alginate based beads of theophylline by the ionic cross-linking technique using different excipients like Eudragit L 100, Sodium carboxy methyl cellulose (SCMC), Sodium lauryl sulphate (SLS), Sodium starch glycolate (SSG), Hydroxy propyl methyl cellulose (HPMC) 5 cps, Polyethylene glycol (PEG) 4000 were used to fabricate Theophylline-alginate beads 
and their effect on drug release were investigated. Since alginate gel can easily be formed by this ionic interaction in aqueous medium, gel beads are commonly obtained by dropping solutions of alginate into solution of electrolyte. ${ }^{2}$

The use of Eudragit L 100, a copolymer based on methacrylic acid and methacrylic acid methyl ester, in preparing theophylline beads was done successfully. ${ }^{3}$ Sodium lauryl sulphate is used as dispersing agent and sodium carboxy methyl cellulose is an anionic water soluble polymer. Their effect on beads showed modified release of theophylline. Sodium starch glycolate absorbs water rapidly, resulting in swelling which leads to rapid disintegration of beads. Hydroxy propyl methyl cellulose and polyethylene glycol can be formed to transparent, tough, flexible film that can reliably control drug release. ${ }^{4,5,6}$

The present study deals with the use of various excipients in theophylline-alginate beads prepared by ionic cross linked technique to observe the in vitro release characteristics, swelling study and surface morphology.

\section{MATERIALS AND METHOD}

Theophylline anhydrous (Eskayef Bangladesh Ltd), Sodium Alginate (BDH Chemicals Ltd., England), Calcium chloride (Merck, Germany), Eudragit L 100 (Merck, Germany), Hydroxy propyl methyl cellulose 5 cps (Merck, Germany), Polyethylene glycol 4000 (Merck, Germany), Sodium carboxy methyl cellulose (Merck, Germany), Sodium lauryl sulphate (Merck, Germany), Sodium starch glycolate (Merck, Germany), used in this study were obtained from the indicated sources.

Preparation of alginate beads by ionic cross linking technique. Sodium alginate solution was prepared by dissolving in distilled water with gentle heat and agitation to have homogenous dispersion. Theophylline and different excipients were added to alginate solution. Calcium chloride $\left(\mathrm{CaCl}_{2}\right)$ solution was prepared as $5 \%$ solution. The solution was continuously stirrer with glass rod while heating in the thermostatic water bath until clear solution appeared. Heating was done up to 30-40 minutes. When all the ingredients were mixed completely a gel was formed, then it was taken in a 23 gauze hypodermic needle fitted with $10 \mathrm{ml}$ syringe and was added drop wise to $5 \%$ calcium chloride solution.

Upon addition, the solution was being mechanically stirred using stirrer. The distance of nozzle from the solution was about $5 \mathrm{~cm}$. When the gel was poured into the electrolyte solution as droplets, they became spherical and it became hard since the temperature was below the gelling point of alginate. Thus alginate beads were prepared. The prepared beads were allowed to stand in the solution for 15 minutes, and then the beads were decanted, washed with distilled water, dried in air for $36-48 \mathrm{hr}^{7,8}$

\section{Characterization of theophylline loaded alginate beads}

Surface morphology: Scanning Electron Microscope (SEM) was used to study the morphology of the prepared beads. Scanning electron microscopy was performed using Hitachi (Model: S-3400 N, Japan) scanning electron microscope having different magnifications and the micrographs are presented in Figure 1 to 8. Prior to examination the beads were placed on carbon tape and then placed on a disk to examine the surface.

Swelling study: The extent of swelling was measured in terms of \% weight gain by the beads. The swelling behaviors of all the formulations were studied. In this test $20 \mathrm{mg}$ of beads from each formulation was kept in petridish containing distilled water. At the end of 1 hour, the beads were withdrawn, soaked with tissue paper and weighed. Then for every 1 hour, weights of beads were noted and the process was continued till the end of 8 hours, \% weight gain by the beads was calculated by the following formula. ${ }^{9}$

Swelling Index $\left.\left.(\mathrm{SI})=\left\{\mathrm{W}_{\mathrm{t}}-\mathrm{W}_{0}\right\} / \mathrm{W}_{0}\right)\right] \times 100$

Here, $\mathrm{W}_{\mathrm{t}}=$ Mass of swollen beads at time $\mathrm{t}$

$\mathrm{W}_{0}=$ Mass of dry beads at $\mathrm{t}=0$ 
In vitro dissolution study: In-vitro dissolution studies were carried out using USP XXX apparatus Type-II (Basket Apparatus) in $900 \mathrm{ml}$ distilled water of $37 \pm 0.5^{\circ} \mathrm{C}$ at a rotational speed of $100 \mathrm{rpm}$. Dissolution samples were withdrawn at appropriate intervals up to 8 hours and then filtered through $0.45 \mu \mathrm{m}$ filters. The drug content was determined spectrophotometrically at $\lambda_{\max }=271 \mathrm{~nm}$ in the filtrate either directly or after appropriate dilution with the dissolution media. The dissolution study for each batch was performed in triplicate.

Kinetic models: The suitability of several equations that are reported in the literature to identify the mechanisms for the release of theophylline was tested with respect to the release data. The data were evaluated according to the following equations: ${ }^{10-13}$

Zero-order equation:

$\mathrm{Q}_{\mathrm{t}}=\mathrm{K}_{0} \mathrm{t}$

Higuchi equation based on Fickian diffusion:

$\mathrm{Q}_{\mathrm{t}}=\mathrm{K}_{\mathrm{H}} \sqrt{ } \mathrm{t}$
Where, $Q$ is the amount of drug release in time $\mathrm{t}, k_{0}$, and $k_{H}$ are rate constant of zero order and Higuchi rate equations respectively.

First order model:

$\log C=\log C_{o}-k t / 2.303$

Where, $\mathrm{C}=$ cumulative percent of drug release, $\mathrm{Co}=$ the initial concentration of drug and $\mathrm{k}$ $=$ first order rate constant.

Determining the correlation coefficient assessed fitness of the data into various kinetic models. The rate constants, for respective models were also calculated from slopes.

\section{RESULTS AND DISCUSSION}

Surface morphology. The drug release from Eudragit L 100 is due to formation of pores and channels and due to swelling of polymer up to some extent. The resultant beads were more spherical and had a smooth surface. ${ }^{14}$ Surface morphology also attributes to this fact in Figure 1 and 2 .

Table 1. Different formulations of alginate beads prepared with various excipients

\begin{tabular}{ccccl}
\hline $\begin{array}{c}\text { Batch } \\
\text { code }\end{array}$ & $\begin{array}{c}\text { Theophylline } \\
(\mathrm{gm})\end{array}$ & $\begin{array}{c}\text { Sodium alginate } \\
(\mathrm{mg})\end{array}$ & Polymer 2 (mg) & Polymer name \\
\hline 1 & 1 & 900 & 100 & Eudragit L 100 \\
2 & 1 & 900 & 100 & Sodium carboxy methyl cellulose \\
3 & 1 & 900 & 100 & Sodium lauryl sulphate \\
4 & 1 & 900 & 100 & Sodium Starch Glycolate \\
5 & 1 & 900 & 100 & HPMC 5 cps \\
6 & 1 & 900 & 100 & Polyethylene glycol 4000 \\
\hline
\end{tabular}

Table 2. Data for swelling index of alginate based theophylline beads with various excipients

\begin{tabular}{ccccccc}
\hline Time(hr) & $\begin{array}{c}\text { F 1(Eud L } \\
100)\end{array}$ & F 2 (SCMC) & F 3 (SLS) & F 4 (SSG) & F 5 (HPMC & F 6 (PEG \\
& 0 & 0 & 0 & 0 & 0 & $4000)$ \\
\hline 0 & 2.99 & 3.10 & 1.85 & 8.60 & 1.67 & 0.53 \\
1 & 5.52 & 4.52 & 3.44 & 9.93 & 3.00 & 3.56 \\
2 & 10.74 & 5.52 & 5.19 & 9.37 & 4.67 & 5.21 \\
3 & 7.98 & 6.86 & 6.04 & 8.61 & 8.67 & 4.56 \\
4 & 5.22 & 9.66 & 7.41 & 8.44 & 5.00 & 3.26 \\
5 & 4.22 & 5.10 & 7.78 & 8.27 & 3.67 & 1.95 \\
6 & 3.68 & 2.76 & 8.15 & 7.29 & 2.67 & 0.98 \\
7 & - & - & 6.67 & 5.96 & 1.67 & 0.33 \\
8 & & & & & &
\end{tabular}

SEM of Formulation 3 with SLS shows that the surface texture is rough compared with Na-CMC. The dense network of drug-polymer-SLS increases the tortuisity, as evident from Figure 4 thus delaying the release of the drug and retarding the penetration of water required to make the beads swell for 
disintegration. Surface morphology of beads with sodium starch glycolate (Formulation 4) reveals that drug crystal is on the surface (Figure 5). SSG is used to control the release of drugs and the size of the beads. From SEM photomicrographs (Figure 6), we can interpret that in F 6 (PEG 4000) the surface morphology is visualized as dense structure. As a result the extent of drug release is only $26 \%$ at the end of 8 hours.

Table 3. R-squared values and release rate of different kinetic models of the formulations

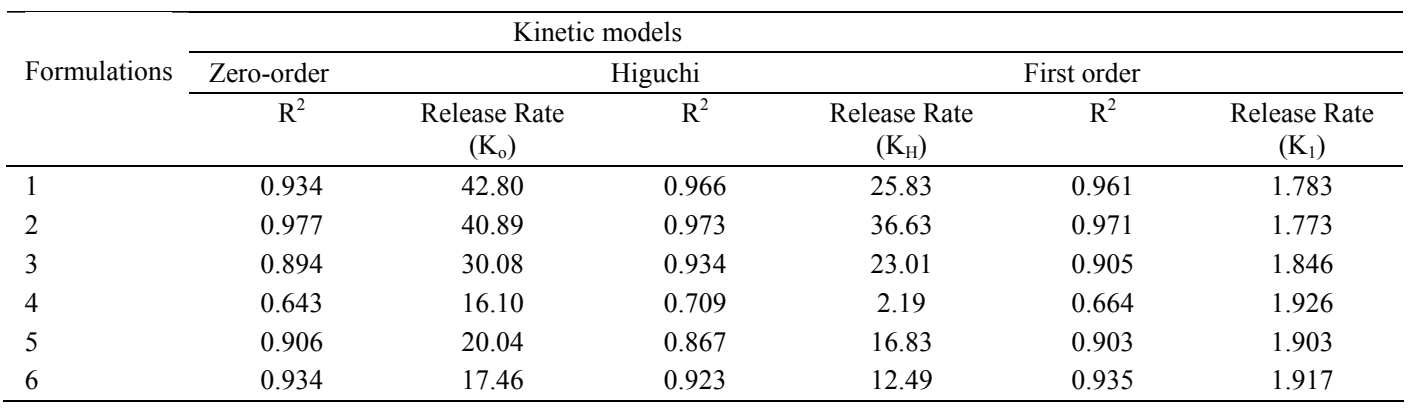
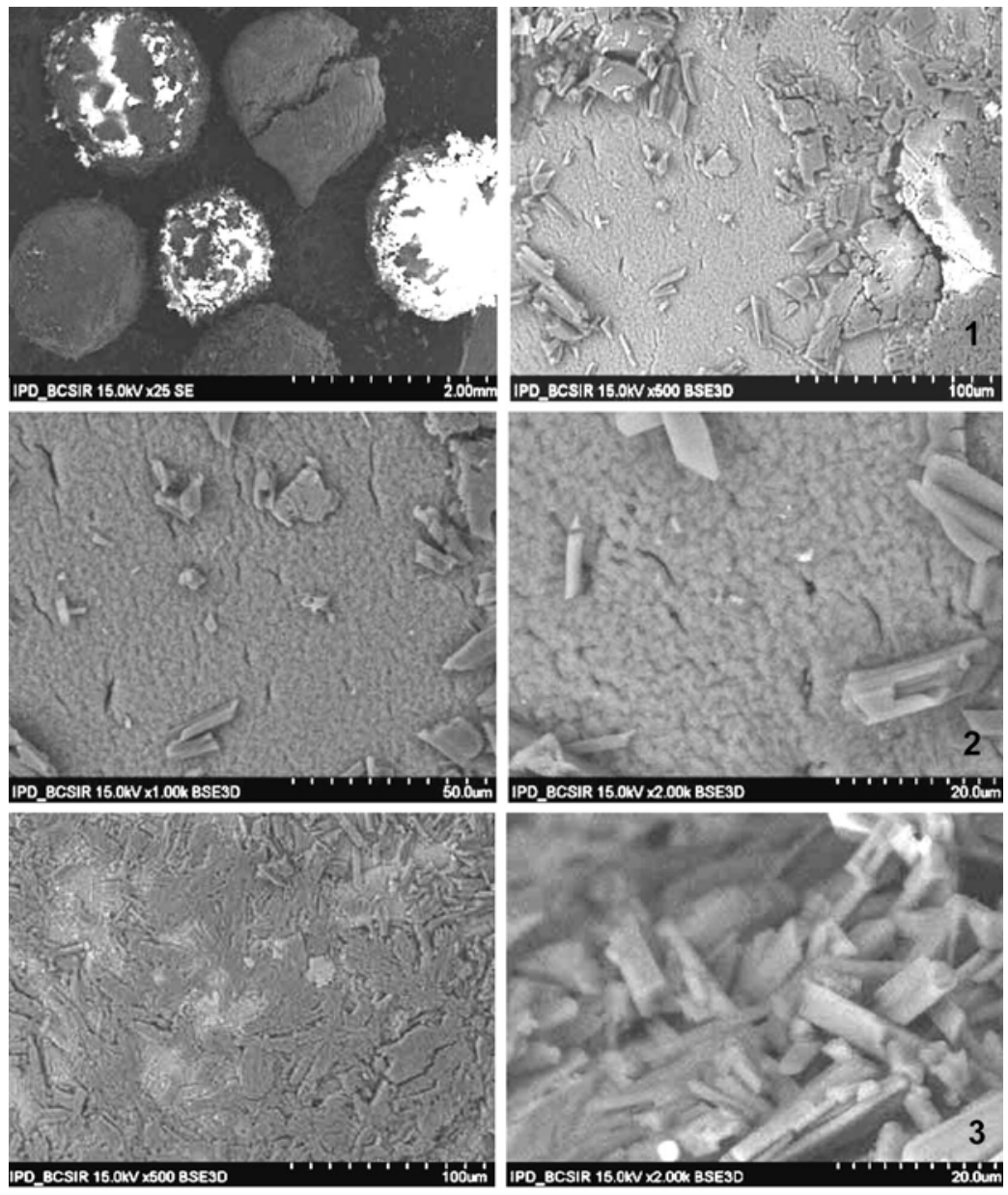

Figs. 1-3: 1. Scanning electron microscopy photomicrographs of formulation 1 at X25 and X500. 2. Scanning electron microscopy photomicrographs of formulation 1 at X1000 and X2000. 3. Scanning electron microscopy photomicrographs of formulation 2 at X500 and $\mathrm{X} 2000$. 

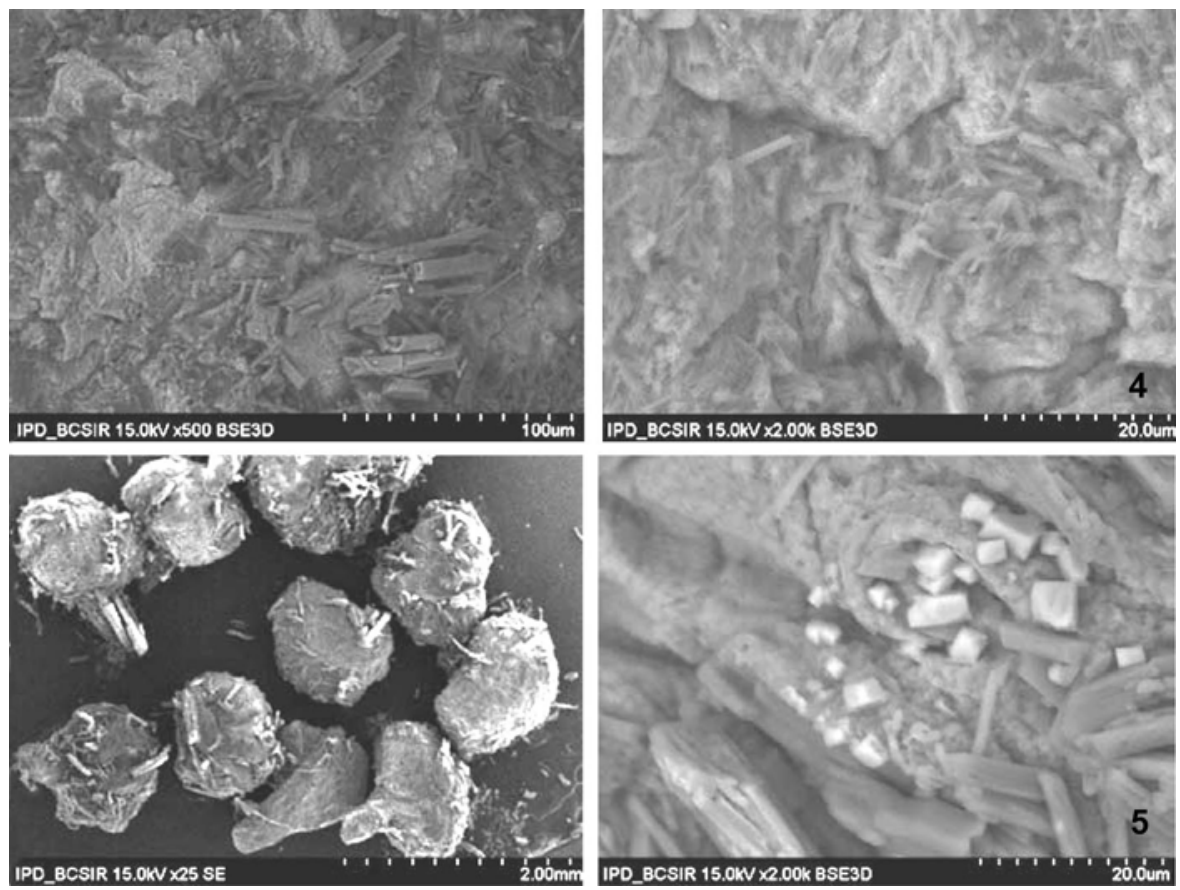

Figs. 4-5: 4: Scanning electron microscopy photomicrographs of formulation 3 at X500 and X2000. 5. Scanning electron microscopy photomicrographs of formulation 4 at X25 and X2000
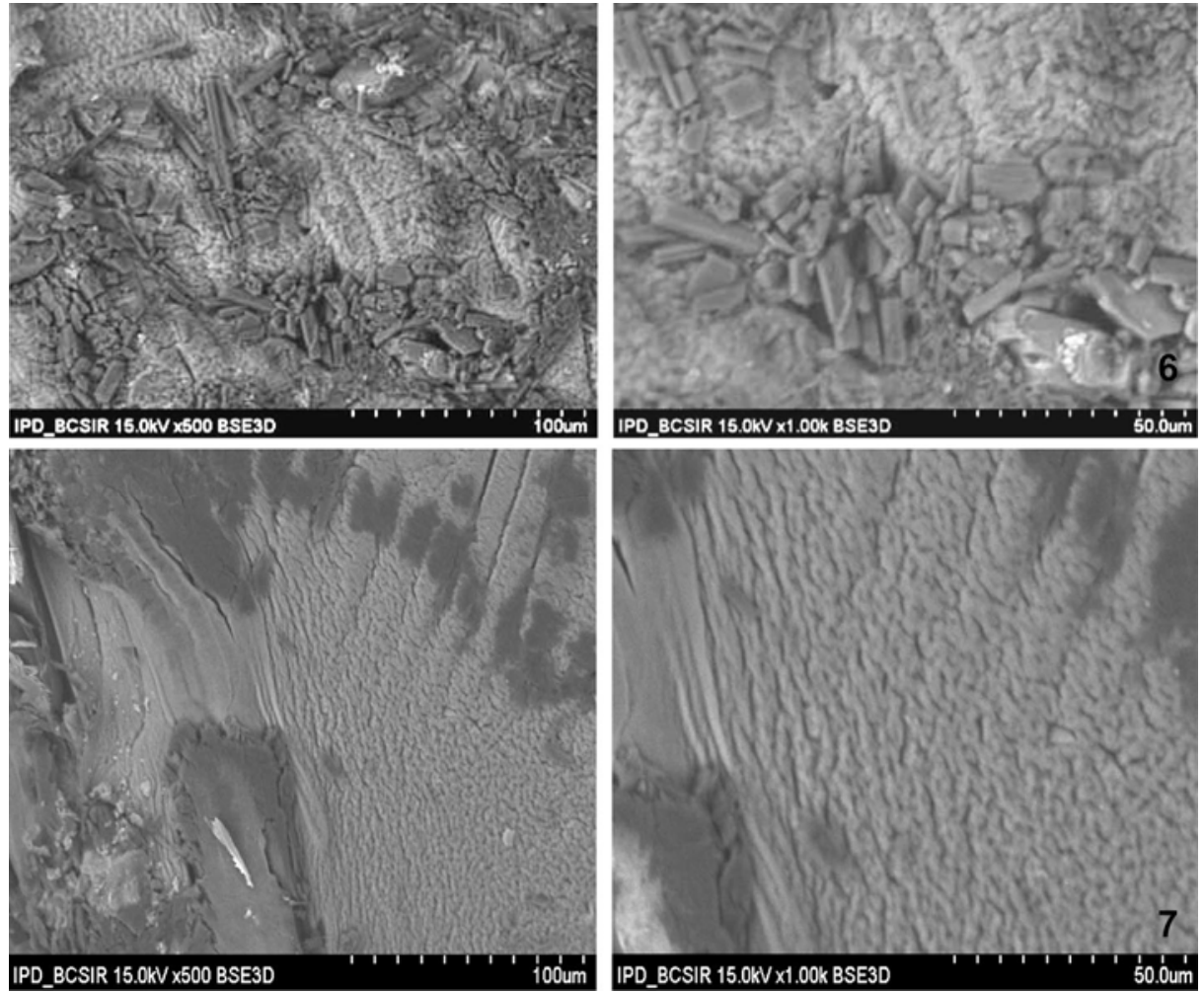

Figs. 6-7: 6. Scanning electron microscopy photomicrographs of formulation 5 at X500 and X1000. 7. Scanning electron microscopy photomicrographs of formulation 6 at X500 and X1000 
Swelling study. Swelling study of alginate based theophylline beads with different excipients prepared in $\mathrm{CaCl}_{2}$ solution was carried out. The formulations were prepared using drug: alginate: excipient in $1 \mathrm{~g}$ : $900 \mathrm{mg}$ : $100 \mathrm{mg}$ ratio. Beads in F 1 contain Eudragit L 100, which is resistant to gastric juice but dissolves readily at above $\mathrm{pH}$ 5.5. They are used to control the active substance from the matrix. In water media it swells highest at 3 hours with SI of $10.74 \%$ (Table 2). Sodium carboxy methyl cellulose containing $F$ beads showed highest swelling at 5th hour (9.66 \%). F 3 swelled high up to $7^{\text {th }}$ hour $(8.15 \%)$. F 4 contains sodium starch glycolate that is used as disintegrant in formulations. It has enormous swelling capacity through rapid water uptake. In this study, the beads swelled high up to $9.93 \%$ within 2 hours. Its enormous swelling helps to disintegrate the beads for long hours after the drug intake time. Hydroxy propyl methyl cellulose 5 cps swelled high up to 4 hours with SI of $8.67 \%$ (F 5). Polyethylene glycol is hydrophilic in nature and their viscosity increases with increasing molecular weight. PEG 4000 (F 6) is used as controlled release agent, which shows $5.21 \%$ swelling at 3 hours (Table 2). The comparison of swelling indices of theophylline beads with various excipients is shown in Figure 8.

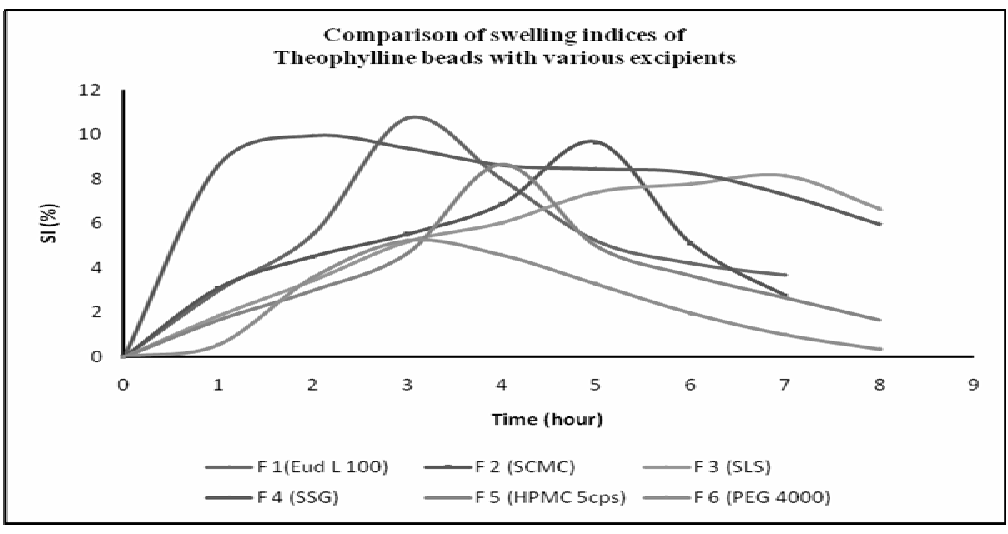

Figure 8. Comparison of swelling indices of theophylline beads with various excipients

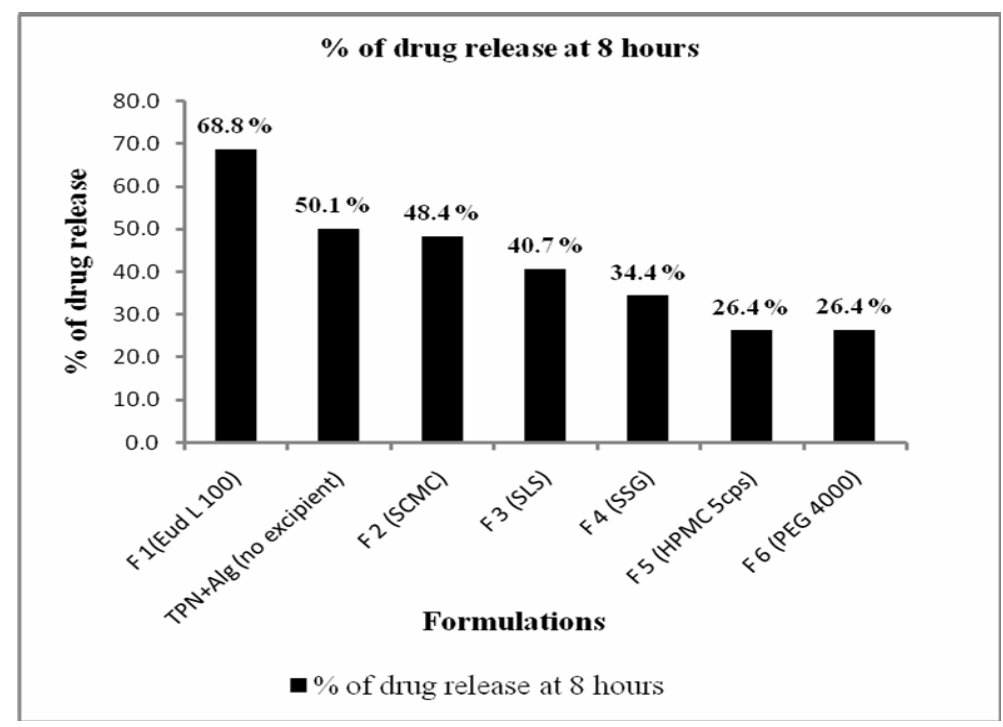

Figure 9. Percent of drug released from the formulations at 8 hours 
In vitro Release Kinetics. When a hydrophilic matrix comes into contact with an aqueous medium, it absorbs water, hydrates and swells to form a gel through which the dissolved drug diffuses out. In case of water soluble polymers, dissolution of the polymer results in a gradual erosion of this gel layer. However, at higher concentrations, the polymer chains entangle to a greater degree culminating in "virtual cross-linking" and therefore formation of a stronger gel layer. Eudragit L 100 was used to prepare beads which give about $69 \%$ (Figure 9) of drug release up to 8 hours. Within 1.5 hours about 40 $\%$ of drug was released. The drug release from Eudragit L 100 is due to formation of pores and channels and due to swelling of polymer up to some extent. Surface morphology also attributes to this fact in Figures 1 and 2. Sodium carboxy methylcellulose (SCMC) is water soluble sustained release polymer, which shows about $49 \%$ drug release at 8 hour. Sodium lauryl sulphate and sodium starch glycolate releases about $40.7 \%$ and $34.4 \%$ of theophylline (Table 3 ). The drug release amount is nearly same for formulation with HPMC 5 cps beads and PEG 4000. HPMC is a hydrophilic polymer used to retard the release of drugs from the beads. Correlating with formulation 4 reveals that addition of polymers can modify the release of theophylline. From Table 3 it was found that $\%$ release of beads containing only theophylline and alginate was $50.12 \%$, and when polymers are used along with alginate the release characteristics were fairly modified. The release data obtained were fitted into different kinetic models (Table 3).

\section{CONCLUSION}

Theophylline loaded alginate beads containing various excipients were prepared successfully by ionic cross linking technique. Apart from the natural water soluble polymer, namely, sodium alginate, the use of different excipients further prolongs the release of the drug. Polymethacrylate Eudragit L 100 showed maximum prolongation of drug release. Hence, further studies can be extended taking
Eudragit L 100 and sodium carboxy methyl cellulose as the release controlling copolymer. The dense network of drug-polymer-excipient delays the release of the drug and retards the penetration of water required to make the beads swell for disintegration. More extensive study can be done when excipients are used to obtain regular and spherical beads, their effect on drug release should be investigated.

\section{ACKNOWLEDGEMENT}

The authors is very obliged to Eskayef Pharmaceuticals Ltd, Bangladesh for providing generously the sample of theophylline anhydrous and other raw materials. The authors also thank Bangladesh Council of Scientific and Industrial Research (BCSIR) laboratories, Dhaka for allowing access to the Scanning Electron Microscope (SEM).

\section{REFERENCES}

1. Ahmed. A. El-Zatahry, Emdad. A. Soliman, El-Sayed A. Hassan, Mohamed. S. Mohy Eldin.2006. Preparation and in vitro release of Theophylline loaded sodium alginate microspheres. ASTF-Scientific Research Outlook Conference. Page\# 155, Category [T6-4] Basic sciences.

2. Sung-Joo Hwang, Gye Ju Rhee, Ki Myung lee, Kyoug-Hee Oh, Chong-Kook Kim.1995. Release characteristics of Ibuprofen from excipient-loaded alginate gel beads. Intl. $J$. Pharm. 116, 125-128.

3. Khan, G.M. 2001. Controlled Release Oral Dosage Forms: Some recent advances in matrix type drug delivery systems. The Sci. 1, 350-354.

4. Formulate excipients- Sodium starch glycolate. Available at http://www.pformulate.com/nastgly.htm.

5. British Pharmacopoeia (B.P.) 2007.London, UK: Stationary Office London.

6. Raymond C Rowe, Paul, J. Sheskey, Marian E Quinn. Handbook of Pharmaceutical Excipients. Sixth edition.

7. Nimmi, I, Chowdhury, JA, Dulal, MM. and Reza, M.S. 2005. Effects of electrolytes on Diclofenac sodium from Agarose beads. Dhaka Univ. J Pharm Sci. 4, 117-120.

8. Pornsak Sriamornsak, Kanokporn Burapapadh, Satit Puttipipatkhachorn and Jurairat Nunthanid. 2008. Effect of aidic mdium on selling and rlease bhaviors of chitosanreinforced calcium pectinate gel beads. Silpakorn U. Science \& Tech. J. 2, 37-44. 
9. Yeole, P.G., Galgatee, U.C., Babla, I.B. and Nakhat. P.D. 2006. Design and evaluation of Xanthan gum based sustained release matrix tablets of Diclofenac Na. Indian J Pharm Sci. 68, $185-189$

10. Donbrow, M. and Samuelov Y. 1980. Zero order drug delivery from double-layered porous films: release rate profiles from ethylcellulose, hydroxypropylcellulose and polyethylene glycol mixtures. J Pharm Pharmacol. 32, 463470 .

11. Higuchi, T. 1961. Rate of release of medicaments from ointment bases containing drugs in suspension. J Pharm. Sci. 50, 874-875.
12. Higuchi,T. 1963. Mechanism of sustained-action medication: theoretical analysis of rate of release of solid drugs dispersed in solid matrices. J Pharm. Sci. 52, 1145 -1149.

13. Merchant, H.A., Shoaib, H.M., Tazeen, J. and Yousuf, R.I. 2006. Once-Daily tablet formulation and in vitro release evaluation of Cefpodoxime using Hydroxypropyl Methylcellulose: A Technical note. AAPS PharmSci Tech. $\mathbf{7}$ Article 78.

14. Morishita, I., Morishita, M., Machida, Y. and Nagai, T.1991. Controlled release microspheres based on Eudragit L100 for the oral administration of erythromycin. Drug Design and Delivery July 7, 309-19. 